\title{
Evaluation of Right Ventricular Function in Patients with Severe and Very Severe Mitral Stenosis
}

\author{
Leila Bigdelu ${ }^{1}$, Morteza Boskabady ${ }^{1}$, Kasra Molooghi ${ }^{1}$, Leila Amirbeik ${ }^{1}$, Maliheh \\ Dadgarmoghaddam ${ }^{1}$, and Ali Azari ${ }^{1}$ \\ ${ }^{1}$ Mashhad University of Medical Sciences
}

April 17, 2021

\begin{abstract}
Background: Right Ventricular (RV) failure has a critical role in the onset and progression of clinical symptoms and the prognosis of patients with Mitral Stenosis (MS), but the exact role, effect, and pathophysiology of RV dysfunction in MS is still controversial. In this study, we aimed to evaluate echocardiographic signs of systolic RV dysfunction using Tissue Doppler Imaging (TDI) and Velocity Vector Imaging (VVI) in subjects with severe and very severe MS. Methods: 46 isolated MS cases (23 severe and 23 very severe) and 23 healthy controls were enrolled in this study. RV function was assessed by tricuspid annular plane systolic excursion (TAPSE), fractional area change (FAC), and peak systolic velocity of the tricuspid annulus (S') using TDI, and RV free wall strain (RVFWS) employing VVI. Furthermore, these values were compared with mitral valve area (MVA) and pulmonary arterial pressure (PAP). Results: TAPSE, FAC, S', and RVFWS values were substantially declined in MS cases compared with healthy controls. Moreover, PAP values were considerably increased in MS subjects. Additionally, S' and TAPSE values were significantly lower in very severe MS patients compared with severe patients. Conclusion: RV systolic function deteriorates in patients with severe and very severe MS. It appears that the degree of the severity of MS can proportionately affect the extent of RV dysfunction and some of its echocardiographic markers such as S' and TAPSE. TDI and VVI can be used as practical early diagnostic methods for RV dysfunction in MS.
\end{abstract}

\section{Title}

Evaluation of Right Ventricular Function in Patients with Severe and Very Severe Mitral Stenosis

\section{Running Head}

RV dysfunction in MS

\section{Authors}

Leila Bigdelu ${ }^{1}$ MD, Morteza Boskabady ${ }^{2}$ MD, Kasra Molooghi ${ }^{3}$ MD, Leila Amirbeik ${ }^{3}$ MD, Maliheh Dadgarmoghaddam $^{4}$ MD, Ali Azari*1 MD

\section{Affiliations}

${ }^{1}$ Vascular and Endovascular Surgery Research Center, Mashhad University of Medical Sciences, Mashhad, Iran

2 Department of Cardiology, Mashhad University of Medical Sciences, Mashhad, Iran

${ }^{3}$ School of Medicine, Mashhad University of Medical Sciences, Mashhad, Iran

${ }^{4}$ Department of Community Medicine, School of Medicine, Mashhad University of Medical Sciences, Mashhad, Iran 


\title{
*Corresponding Author Information
}

Ali Azari, MD, Assistant Professor of Cardiovascular surgery in Ghaem Hospital, Mashhad University of Medical Sciences, Mashhad, Iran.

Postal Address: Ghaem Hospital, Koohsangi Street, Shariati Square, Mashhad, Razavi Khorasan Province, Mashhad, Iran. / Postal Code: 9176699616

Email:aliazariii.id@gmail.com/ Phone 8 Fax Number: +985138012739

ORCID :0000-0003-0953-9523

\section{Funding}

None

\begin{abstract}
Background: Right Ventricular (RV) failure has a critical role in the onset and progression of clinical symptoms and the prognosis of patients with Mitral Stenosis (MS), but the exact role, effect, and pathophysiology of RV dysfunction in MS is still controversial. In this study, we aimed to evaluate echocardiographic signs of systolic RV dysfunction using Tissue Doppler Imaging (TDI) and Velocity Vector Imaging (VVI) in subjects with severe and very severe MS.
\end{abstract}

Methods: 46 isolated MS cases (23 severe and 23 very severe) and 23 healthy controls were enrolled in this study. RV function was assessed by tricuspid annular plane systolic excursion (TAPSE), fractional area change (FAC), and peak systolic velocity of the tricuspid annulus (S') using TDI, and RV free wall strain (RVFWS) employing VVI. Furthermore, these values were compared with mitral valve area (MVA) and pulmonary arterial pressure (PAP).

Results: TAPSE, FAC, S', and RVFWS values were substantially declined in MS cases compared with healthy controls. Moreover, PAP values were considerably increased in MS subjects. Additionally, S' and TAPSE values were significantly lower in very severe MS patients compared with severe patients.

Conclusion: RV systolic function deteriorates in patients with severe and very severe MS. It appears that the degree of the severity of MS can proportionately affect the extent of RV dysfunction and some of its echocardiographic markers such as S' and TAPSE. TDI and VVI can be used as practical early diagnostic methods for RV dysfunction in MS.

\section{Keywords (MeSH)}

Mitral Valve Stenosis; Echocardiography; Right Ventricle; Velocity vector imaging; Tissue Doppler imaging; Strain rate imaging

\section{Introduction}

Mitral stenosis (MS) is a cardiac disorder generally caused by Rheumatic Fever (RF) and characterized by morphological changes in the mitral valve such as commissural fusion, retraction, thickening and narrowing of the mitral valve apparatus and leaflets ${ }^{1}$. Right Ventricular (RV) failure has a critical role in the onset and progression of clinical symptoms and the prognosis of MS patients, but the exact role, effect, and pathophysiology of RV dysfunction in MS is still controversial ${ }^{2}$.. Some theories suggest that the pathophysiological and structural changes that are caused by MS lead to pulmonary hypertension $(\mathrm{PH})$ and increased pulmonary artery pressure (PAP), which eventually result in RV dysfunction ${ }^{3}$. It is still not known whether the degree of MS severity has a correlation with RV dysfunction independent of PH and high PAP.

Furthermore, echocardiographic markers are able to predict rehospitalization rates in MS patients ${ }^{4}$. Since a delayed diagnosis can result in high rates of morbidity and mortality, while early interventions have immensely improved clinical outcomes, early detection of RV dysfunction is critical for the patients ${ }^{5}$. Various imaging methods have been employed to evaluate RV function such as radionuclide angiography, contrast 
right ventriculography, and novel modalities like magnetic resonance imaging; however, most are confined by restrictions ${ }^{6}$. Although cardiac imaging has remarkably improved in recent years, measuring the size and function of RV has remained a complex process due to the special crescent structure of the RV and morphological differences between the Left Ventricle (LV) and RV ${ }^{7-9}$. Tissue Doppler imaging (TDI) is a simple, safe, cost-effective, and accurate modality for detecting both RV and LV dysfunction ${ }^{10}$. Even though TDI enhanced the diagnostic yield of echocardiography, the reliability of its data is controversial due to its angle dependency ${ }^{11}$. Velocity Vector Imaging (VVI) is among the more novel and more reliable means of investigating LV and RV function using strain imaging, due to being angle-independent ${ }^{12-16}$.

In order to assess the correlation of the degree of MS severity with RV dysfunction and to discover the echocardiographic markers that can be used as early diagnostic features of subclinical RV dysfunction, we aimed to investigate the echocardiographic signs of systolic RV dysfunction using TDI and VVI in patients with severe and very severe mitral stenosis.

\section{Methods and Materials}

\section{Subjects}

This cross-sectional study was performed on 46 patients with isolated MS and 23 healthy controls (matched by sex and age) at the Echocardiography Center of Qaem Hospital, Mashhad, Iran. Patients who had a history of more-than-moderate mitral insufficiency, LV failure, Ejection Fraction (EF) of $<50 \%$, valvular surgery, stenosis or insufficiency in other cardiac valves, were not included in the study. Additionally, patients with a history of atrial fibrillation (AF), underlying respiratory disease, coronary artery disease (CAD), or smoking were excluded before the start of the study. According to the latest guideline (at the time of the study) for the management of patients with valvular heart disease, patients were categorized into two groups of 23 severe (mitral valve area (MVA) between 1-1.5 cm) and 23 very severe MS subjects (MVA [?]1.0 cm) 2 .

\section{Echocardiographic Assessments and Outcome Measurements}

Patients underwent transthoracic echocardiography using a Siemens ACUSON SC2000 by an expert specialist of echocardiography. Measurements were in accordance with the latest guideline of The American Society of Echocardiography and the European Association of Cardiovascular Imaging ${ }^{9}$. All routine echocardiographic parameters in MS patients were measured and calculated using standard parasternal and apical windows. The planimetric method was utilized to measure MVA. Additionally, RV size was evaluated by conventional apical four-chamber view at end-diastole. RV systolic function was assessed by tricuspid annular plane systolic excursion (TAPSE), fractional area change (FAC), peak systolic velocity of the tricuspid annulus (S') using TDI, and RV free wall strain (RVFWS) by VVI software Syngo® Workplace. RV longitudinal function was evaluated by TAPSE in M-mode echocardiography with the cursor optimally aligned along the direction of the lateral annulus of tricuspid valve in the apical four-chamber cardiac view. TDI was employed to calculate S', while taking caution to prevent velocity underestimation by keeping the annulus and the basal segment aligned with the Doppler cursor. FAC was assessed in RV-focused apical four-chamber view, by determining the end-diastolic and end-systolic area $(100 \times$ [End-diastolic area - End-systolic area] / Enddiastolic area). Recorded offline images were analyzed by Syngo $\mathbb{R}$ Workplace software in order to measure strain. To guarantee that the entire myocardial wall was included, endocardial and epicardial borders of RV were traced and adjusted manually. The software automatically traced and calculated the velocity of the tangential motion of the speckles of the myocardium. RV free wall (RVFW) was defined as apical lateral, mid lateral, and basal lateral segments; and RV free wall strain (RVFWS) values were recorded and reported for each patient. (Figure 1.)

\section{Statistical Analysis}

Statistical analyses were carried out using the SPSS for Windows TM, version 16.0 software packages. Measures of central tendency, dispersion, and frequency distribution were used for data description. In order to compare RV functional parameters the analysis of variance (ANOVA) or its non-parametric equivalent, 
Kruscal-wallis test was used. The Post-Hoc Tukey test was utilized to compare the RV echocardiographic parameters between multiple groups and the Chi-square test was used for qualitative variables. In addition, Pearson or Spearman's correlation tests were used to assess the relation between MVA and RV function parameters. A two-sided $\mathrm{P}$-value $<0.05$ was considered statistically significant.

\section{Results}

Forty-six MS patients (23 severe and 23 very severe) and 23 healthy controls were recruited into our study. The mean age of the participants was $42.49 \pm 13.1$ years old (ranging from 19 to 74 years) and $68.1 \%(\mathrm{n}=47$ ) of the subjects were female. The demographic information of the participants are summarized in table 1. Mean FAC was significantly higher in healthy subjects compared with MS patients $(\mathrm{p}<0.001)$. However, no meaningful differences were observed between severe and very severe MS patients $(\mathrm{p}=0.97)$. Table 2 shows the comparison of RV functional parameters between the study groups. There were significant differences in $\mathrm{S}$ ' values within groups $(\mathrm{p}=0.008)$ using one-way ANOVA test. Further analysis by Post-Hoc Tukey test showed that $S^{\prime}$ was significantly lower in patients with very severe MS compared with severe MS cases $(\mathrm{p}=0.017)$ and healthy individuals $(\mathrm{p}=0.012$, table 2$)$. There was a gradual decline in TAPSE value from the healthy subjects to very severe MS patients $(\mathrm{p}<0.001$, table 2$)$. Furthermore, RVFWS was significantly higher in healthy subjects in comparison with severe and very severe MS patients (27.4 \pm 2.7 vs. $13.6 \pm 7.2$ and $13.4 \pm 5.2$, respectively; $\mathrm{p}<0.001)$. Average PAP values were considerably higher in all MS patients, but displayed no discrepancies among severe and very severe subjects.

Pearson and Spearman's correlation tests were used to investigate possible associations of MVA or PAP values with other echocardiographic parameters. MVA values showed significant correlation with TAPSE and S' values in patients with MS $(\mathrm{r}=0.55$ and $\mathrm{r}=0.43, \mathrm{p}<0.001$ and $\mathrm{p}=0.004$, respectively; shown in table $3)$. Moreover, the correlation analysis between PAP and other factors showed a substantial connection with FAC and RVFWS values $(\mathrm{p}<0.05)$.

\section{Discussion}

Our findings confirm that RV systolic function was diminished in MS patients. S', FAC, TAPSE, and RVFWS values were significantly reduced in MS cases compared with the control group. Furthermore, average PAP values were substantially increased in MS patients. In addition, S' and TAPSE values were considerably lower in very severe MS patients compared with severe patients, which not only verify the relationship between MS and RV dysfunction, but also indicate that the severity of MS correlates with the extent of RV dysfunction and its echocardiographic markers.

Various imaging modalities have been utilized in order to diagnose MS patients with pre-symptomatic and subclinical RV failure. Tanboga et al. employed TDI and two-dimensional speckle tracking echocardiography in order to assess $59 \mathrm{MS}$ cases (33 mild and 26 moderate patients) ${ }^{17}$. Even though they found no connections between FAC values of healthy controls and MS patients, markers such as RVFWS and strain rate demonstrated strong evidence of RV dysfunction in MS cases ${ }^{17}$. However, their subgroup analysis on mild and moderate patients did not show any correlations between the severity of the disease and the level of RV dysfunction. It is important to note that Tanboga et al. studied mild and moderate MS cases, whereas our study selected severe to very severe patients. The duration and degree of severity of the disease can affect the process of RV dysfunction, which might justify this divergence in findings. Thus, further studies on various degrees of severity and duration of MS with larger samples are required to shed more light on the relation of severity of MS with the degree of RV dysfunction. Similar studies have consistently demonstrated that declined TAPSE, S', and RVFWS values are good predictors of early RV dysfunction but have not discussed the effects of severity of MS 4,6,18,19.

Even though our findings agree with previous studies, the pathophysiology of RV dysfunction is still debatable. Some studies have speculated that the initial rheumatic heart disease and its subsequent scarring process might directly damage the RV myocardium in MS patients, which could possibly lead to RV dysfunction and reduced RV ejection performance ${ }^{20,21}$. On the other hand, according to recent studies, MS patients tend to have a passive elevated left atrial pressure, which results in pulmonary venous hypertension ${ }^{3,22}$. As a 
complication of longstanding MS, a retrograde passive increase in pressure contributes to elevated pulmonary arterial pressure ${ }^{3}$. In time, this phenomenon alongside pulmonary arteriolar vasoconstriction and changes in the pulmonary microvasculature would subsequently lead to pulmonary artery hypertension, which increases RV afterload and results in RV dysfunction and right sided heart failure ${ }^{3,22}$. A study by Ozdemir et al. provided evidence for the latter hypothesis by examining RV systolic function in 45 mild to moderate pure MS patients against 21 healthy individuals ${ }^{6}$. Their results demonstrated that global RV strain and S' are decreased in MS patients. They divided global RV strain into two regional components of interventricular septum (IVS) and RVFWS and showed that the rheumatic process does not influence the contractility of the RVFWS. In addition, their findings implied that RV dysfunction was more likely due to mild increase in PAP and its subsequent increased afterload. Even though we initially aimed to investigate the correlation of the degree of MS severity and echocardiographic parameters of RV function through multivariate analysis, irrespective of PAP values, our sample size restricted our analysis, and consequently multivariate analysis could not be performed distinctively on severe and very severe subgroups, in order to exclude the effect of PH and PAP. However, our results indicated that PAP has a significant correlation with RVFWS in MS patients, which was similar to the findings of Ozdemir et al. Many studies on PH have proven the correlation of increased PAP with RV dysfunction and have also shown the relation of echocardiographic impairments such as RVFWS with PAP and MS ${ }^{12,23-28}$. Thus, RVFWS can be used as an early indicator of RV dysfunction in MS patients.

$\mathrm{PH}$ in MS patients is potentially reversible. Several studies have shown rapid improvements in echocardiographic variables such as TAPSE, FAC, and RVFWS after interventions such as percutaneous transvenous mitral commisurotomy, balloon mitral valvuloplasty, or mitral valve repair in MS patients ${ }^{29-33}$. Future studies could investigate the possibility of early interventions in MS patients as soon as echocardiographic markers start to deteriorate (before the remodeling of RV and the appearance of clinical signs and symptoms), in pursuit of increased survival and quality of life in these individuals.

\section{Limitations}

At the preliminary stage of designing this study, we aimed to investigate the correlation of the degree of MS severity and echocardiographic parameters of RV function through multivariate analysis, independent of PAP values. However, we were unable to identify and include the sufficient number of patients with various degrees of MS severity (who met our inclusion/exclusion criteria) necessary for providing a significantly meaningful multivariate analysis on severe and very severe subgroups, while accounting for and eliminating the effect of PAP.

\section{Conclusion}

In conclusion, our data verify that RV systolic function is decreased in patients with severe and very severe MS. TDI and VVI echocardiographic features such as S', TAPSE, RVFWS, and FAC are useful predictors of RV dysfunction. We advise regular echocardiographic measurements for MS patients in order to ensure early detection of RV dysfunction predictors. If impairments in these parameters were recognized early in the course of the disease, timely interventions might be able to prevent, decrease, or reverse the adverse effects of MS such as PH or RV dysfunction and drastically improve the prognosis of MS patients. Further studies with larger cohorts of mild, moderate, severe, and very severe MS cases are necessary to establish the extent of RV systolic dysfunction using various echocardiographic measurements.

\section{Declarations}

\section{Funding}

None.

\section{Conflicts of interest/Competing interests}

None

\section{Ethical approval}


This study fully complies with the Declaration of Helsinki and was approved by the Ethics Committee of Mashhad University of Medical Sciences under the project number of 950541 and code of IR.MUMS.fm.REC.1395.566.

\section{Consent to participate}

All participants were informed of the study protocol and signed an informed written consent to take part in this study.

\section{Availability of data and material}

The data underlying this article cannot be shared publicly due to the privacy of individuals that participated in the study. The anonymous data will be shared upon reasonable request to the corresponding author.

\section{Author contributions}

LB: Concept/design, Data collection, Data interpretation, Drafting Article, Critical revision of article, Approval of article

MB: Concept/design, Data collection, Data interpretation, Drafting article, Approval of article

KM: Literature Search, Data analysis, Statistics, Data interpretation, Drafting article, Critical revision of article, Approval of article

LA: Literature Search, Data interpretation, Drafting article, Critical revision of article, Approval of article

MDM: Data analysis, Statistics, Data interpretation, Drafting article, Approval of article

AA: Concept/design, Data interpretation, Drafting Article, Approval of article

\section{Acknowledgements}

The authors would like to acknowledge the support of Department of Heart Surgery and Department of Echocardiography of Ghaem Hospital, Mashhad, Iran, in all the steps of the study.

\section{Submission declaration and verification}

The authors declare that this work is original, has not been published previously, and is not under consideration for publication elsewhere. All authors approve its publication, and if accepted, it will not be published elsewhere in the same form, in English or in any other language, including electronically, without the written consent of the copyright-holder.

\section{References}

1. Feldman T. Rheumatic heart disease. Curr Opin Cardiol.1996;11(2):126-130.

2. Nishimura RA, Otto CM, Bonow RO, et al. 2014 AHA/ACC guideline for the management of patients with valvular heart disease: a report of the American College of Cardiology/American Heart Association Task Force on Practice Guidelines. J Thorac Cardiovasc Surg.2014;148(1):e1-e132.

3. Libby P, Zipes DP. Braunwald's Heart Disease: A Textbook of Cardiovascular Medicine. Elsevier; 2019.

4. Kalkan K, Kalkan F, Aksakal E, et al. Assessment of right ventricular dysfunction in patients with mitral stenosis: A speckle tracking study.J Clin Ultrasound. 2020;48(5):269-274.

5. Baumgartner H, Falk V, Bax JJ, et al. 2017 ESC/EACTS Guidelines for the management of valvular heart disease. Eur Heart J.2017;38(36):2739-2791.

6. Ozdemir AO, Kaya CT, Ozdol C, et al. Two-dimensional longitudinal strain and strain rate imaging for assessing the right ventricular function in patients with mitral stenosis. Echocardiography.2010;27(5):525-533.

7. Badano LP, Muraru D, Parati G, Haugaa K, Voigt J-U. How to do right ventricular strain. European Heart Journal - Cardiovascular Imaging. 2020;21(8):825-827. 
8. Garcia Gigorro R, Renes Carreño E, Mayordomo S, et al. Evaluation of right ventricular function after cardiac surgery: The importance of tricuspid annular plane systolic excursion and right ventricular ejection fraction. J Thorac Cardiovasc Surg. 2016;152(2):613-620.

9. Lang RM, Badano LP, Mor-Avi V, et al. Recommendations for cardiac chamber quantification by echocardiography in adults: an update from the American Society of Echocardiography and the European Association of Cardiovascular Imaging. Eur Heart J Cardiovasc Imaging.2015;16(3):233-270.

10. Yadav H, Unsworth B, Fontana M, et al. Selective right ventricular impairment following coronary artery bypass graft surgery. Eur J Cardiothorac Surg. 2010;37(2):393-398.

11. Pellerin D, Sharma R, Elliott P, Veyrat C. Tissue Doppler, strain, and strain rate echocardiography for the assessment of left and right systolic ventricular function. Heart. 2003;89 Suppl 3(Suppl 3):iii9-17.

12. Rajagopal S, Forsha DE, Risum N, et al. Comprehensive assessment of right ventricular function in patients with pulmonary hypertension with global longitudinal peak systolic strain derived from multiple right ventricular views. J Am Soc Echocardiogr.2014;27(6):657-665.e653.

13. Park JH, Kusunose K, Motoki H, et al. Assessment of Right Ventricular Longitudinal Strain in Patients with Ischemic Cardiomyopathy: Head-to-Head Comparison between Two-Dimensional Speckle-Based Strain and Velocity Vector Imaging Using Volumetric Assessment by Cardiac Magnetic Resonance as a "Gold Standard". Echocardiography. 2015;32(6):956-965.

14. Jung SY, Shin JI, Choi JY, Park SJ, Kim NK. Velocity Vector Imaging Assessment of Functional Change in the Right Ventricle during Transcatheter Closure of Atrial Septal Defect by Intracardiac Echocardiography. J Clin Med. 2020;9(4).

15. Li J, Lin X, Li H, et al. Right ventricular diastolic dysfunction in patients with obstructive sleep apnea syndrome. Echocardiography.2020;37(2):317-322.

16. Meng H, Chandrasekaran K, Villarraga HR, et al. Right and left ventricular interaction in pulmonary hypertension: Insight from velocity vector imaging. Echocardiography. 2019;36(5):877-887.

17. Tanboga IH, Kurt M, Bilen E, et al. Assessment of right ventricular mechanics in patients with mitral stenosis by two-dimensional deformation imaging. Echocardiography. 2012;29(8):956-961.

18. Tayyareci Y, Nisanci Y, Umman B, et al. Early detection of right ventricular systolic dysfunction by using myocardial acceleration during isovolumic contraction in patients with mitral stenosis. Eur $J$ Echocardiogr. 2008;9(4):516-521.

19. Younan H. Detection of subclinical right ventricular systolic dysfunction in patients with mitral stenosis by two dimensional strain and strain rate imaging. The Egyptian Heart Journal.2015;67(1):47-53.

20. Harvey RM, Ferrer I, Samet P, et al. Mechanical and myocardial factors in rheumatic heart disease with mitral stenosis. Circulation. 1955;11(4):531-551.

21. Malhotra V, Beohar PC, Gondal R, Kaul UA, Khanna SK. An autopsy study of rheumatic heart disease. Part II. Associated findings. Jpn Heart J. 1987;28(1):7-14.

22. Nagel E, Stuber M, Hess OM. Importance of the right ventricle in valvular heart disease. Eur Heart J. 1996;17(6):829-836.

23. Fukuda Y, Tanaka H, Sugiyama D, et al. Utility of right ventricular free wall speckle-tracking strain for evaluation of right ventricular performance in patients with pulmonary hypertension. J Am Soc Echocardiogr. 2011;24(10):1101-1108.

24. Yildirimturk O, Helvacioglu FF, Tayyareci Y, Yurdakul S, Aytekin S. Assessment of right ventricular endocardial dysfunction in mild-to-moderate mitral stenosis patients using velocity vector imaging. Echocardiography. 2012;29(1):25-33. 
25. Lu KJ, Chen JX, Profitis K, et al. Right ventricular global longitudinal strain is an independent predictor of right ventricular function: a multimodality study of cardiac magnetic resonance imaging, real time threedimensional echocardiography and speckle tracking echocardiography. Echocardiography. 2015;32(6):966-974.

26. Siddiqui I, Rajagopal S, Brucker A, et al. Clinical and Echocardiographic Predictors of Outcomes in Patients With Pulmonary Hypertension. Am J Cardiol. 2018;122(5):872-878.

27. Theres L, Hübscher A, Stangl K, et al. Associations of 2D speckle tracking echocardiography-based right heart deformation parameters and invasively assessed hemodynamic measurements in patients with pulmonary hypertension. Cardiovasc Ultrasound. 2020;18(1):13.

28. Vitarelli A, Mangieri E, Terzano C, et al. Three-dimensional echocardiography and 2D-3D speckletracking imaging in chronic pulmonary hypertension: diagnostic accuracy in detecting hemodynamic signs of right ventricular (RV) failure. $J$ Am Heart Assoc.2015;4(3):e001584.

29. Khanna R, Raghuvanshi AS, Kumar S, et al. Immediate impact of percutaneous transvenous mitral commisurotomy on right ventricle longitudinal strain in patients of mitral stenosis.Echocardiography. 2018;35(10):1525-1532.

30. Kumar V, Jose VJ, Pati PK, Jose J. Assessment of right ventricular strain and strain rate in patients with severe mitral stenosis before and after balloon mitral valvuloplasty. Indian Heart J.2014;66(2):176-182.

31. Maffessanti F, Gripari P, Tamborini G, et al. Evaluation of right ventricular systolic function after mitral valve repair: a two-dimensional Doppler, speckle-tracking, and three-dimensional echocardiographic study. $J$ Am Soc Echocardiogr.2012;25(7):701-708.

32. Reyes VP, Raju BS, Wynne J, et al. Percutaneous balloon valvuloplasty compared with open surgical commissurotomy for mitral stenosis. N Engl J Med. 1994;331(15):961-967.

33. Shojaeifard M, Samiei N, Firouzi A, et al. Rapid improvement in right ventricular free wall longitudinal strain measures after successful percutaneous mitral commissurotomy. Echocardiography.2019;36(10):18461851.

Tables

Table 1 . Demographic characteristics of patients within subgroups.

\begin{tabular}{|c|c|c|c|c|c|}
\hline Variables & Variables & $\begin{array}{l}\text { Control Group } \\
\mathrm{N}=23 \mathrm{~N}(\%) \text { or } \\
\text { Mean } \pm \mathrm{SD}\end{array}$ & $\begin{array}{l}\text { Patients with } \\
\text { severe MS N=} 23 \\
\mathrm{~N}(\%) \text { or } \\
\text { Mean } \pm \mathrm{SD}\end{array}$ & $\begin{array}{l}\text { Patients with } \\
\text { very severe MS } \\
\mathrm{N}=23 \mathrm{~N}(\%) \text { or } \\
\text { Mean } \pm \mathrm{SD}\end{array}$ & ${ }^{*} \mathrm{P}$-value \\
\hline \multirow[t]{2}{*}{ Sex** } & Male & $9(39.1 \%)$ & $6(28.1 \%)$ & $7(30.4 \%)$ & 0.69 \\
\hline & Female & $14(61.8 \%)$ & $17(71.9 \%)$ & $16(69.5 \%)$ & \\
\hline Age (y) & Age (y) & $36.7 \pm 11.9$ & $43.7 \pm 13.3$ & $47.1 \pm 12.3$ & 0.08 \\
\hline $\mathrm{BSA}\left(\mathrm{m}^{2}\right)$ & $\operatorname{BSA}\left(\mathrm{m}^{2}\right)$ & $1.73 \pm 0.10$ & $1.77 \pm 0.09$ & $1.71 \pm 0.19$ & 0.386 \\
\hline PR (bpm) & $\mathrm{PR}(\mathrm{bpm})$ & $79.5 \pm 12.4$ & $86.1 \pm 10.4$ & $84.4 \pm 14.7$ & 0.331 \\
\hline
\end{tabular}

*ANOVA test was used to compare the variables between groups. ${ }^{* *}$ Chi-square test was used to compare the distribution of gender between study groups.

BSA: Body Surface Area in square meter $\left(\mathrm{m}^{2}\right)$, PR: Pulse Rate in beats per minute (bpm)

Table 2 . Comparison of RV functional parameters between the study groups. 


\begin{tabular}{|c|c|c|c|c|c|}
\hline Variables & $\begin{array}{l}\text { Control Group } \\
\mathrm{N}=23 \text { Mean } \pm \mathrm{SD}\end{array}$ & $\begin{array}{l}\text { Patients with } \\
\text { severe MS N=23 } \\
\text { Mean } \pm \text { SD }\end{array}$ & $\begin{array}{l}\text { Patients with } \\
\text { very severe MS } \\
\mathrm{N}=23 \text { Mean } \pm \mathrm{SD}\end{array}$ & leuro P-value & $\begin{array}{l}\text { P-value between } \\
\text { subgroups }\end{array}$ \\
\hline $\mathrm{PAP}(\mathrm{mmHG})$ & $23.2 \pm 3.3$ & $43.2 \pm 9.3$ & $47.0 \pm 19.1$ & $<0.001$ & $\begin{array}{l}\mathrm{C} \text { vs } 2-^{* * *} \mathrm{C} \text { vs } \\
3-* * * 2 \text { vs } 3-^{*}\end{array}$ \\
\hline FAC $(\%)$ & $52.8 \pm 8.6$ & $27.9 \pm 14.0$ & $29.0 \pm 8.1$ & $<0.001$ & $\begin{array}{l}\mathrm{C} \text { vs } 2-^{* * *} \mathrm{C} \text { vs } \\
3-* * * 2 \text { vs } 3^{*}\end{array}$ \\
\hline $\mathrm{S}^{\prime}(\mathrm{cm} / \mathrm{s})$ & $13.6 \pm 1.9$ & $14.1 \pm 4.8$ & $10.7 \pm 2.1$ & 0.008 & $\begin{array}{l}\mathrm{C} \text { vs } 2-^{*} \mathrm{C} \text { vs } \\
3-* * 2 \text { vs } 3-^{* *}\end{array}$ \\
\hline TAPSE $(\mathrm{cm})$ & $2.5 \pm 0.4$ & $2.3 \pm 0.4$ & $1.6 \pm 0.5$ & $<0.001$ & $\begin{array}{l}\mathrm{C} \text { vs } 2-^{*} \mathrm{C} \text { vs } \\
3-* * * 2 \text { vs } 3 \text { - }^{* * *}\end{array}$ \\
\hline Strain (\%) & $27.4 \pm 2.7$ & $13.6 \pm 7.2$ & $13.4 \pm 5.2$ & $<0.001$ & $\begin{array}{l}\mathrm{C} \text { vs } 22^{* * *} \mathrm{C} \text { vs } \\
3-* * * 2 \text { vs } 3^{*}\end{array}$ \\
\hline
\end{tabular}

PAP: Pulmonary artery pressure, FAC: Fractional Area Change, S: Velocity of the tricuspid annular systolic motion, TAPSE: Tricuspid annular plane systolic excursion

*: $\mathrm{P}>0.05$

$* *: \mathrm{P}<0.05$

$* * *: \mathrm{P}<0.01$

: The Post-Hoc Tukey test

Table 3 . Evaluation of the possible associations between echocardiographic parameters and MVA and PAP values in MS patients.

\begin{tabular}{lllll}
\hline Variables & MVA $^{*}\left(\mathrm{~cm}^{2}\right)$ & MVA $^{*}\left(\mathrm{~cm}^{2}\right)$ & PAP $(\mathrm{mmHg})^{* *}$ & PAP $(\mathrm{mmHg})^{* *}$ \\
\hline & $\mathbf{r}$ & P-value & $\mathbf{r}$ & P-value \\
FAC $(\%)$ & 0.09 & 0.53 & 0.47 & 0.005 \\
S $(\mathrm{cm} / \mathrm{s})$ & 0.43 & 0.004 & 0.15 & 0.39 \\
TAPSE $(\mathrm{cm})$ & 0.55 & $<0.001$ & 0.11 & 0.55 \\
RVFWS $(\%)$ & 0.10 & 0.51 & 0.34 & 0.04 \\
\hline
\end{tabular}

MVA: Mitral Valve Area, PAP: Pulmonary artery pressure, FAC: Fractional Area Change, S: Velocity of the tricuspid annular systolic motion, TAPSE: Tricuspid annular plane systolic excursion, RVFWS: Right Ventricular free wall Strain

*: Pearson's correlation test

**: Spearman's correlation test

\section{Figure}

Figure 1.

Velocity vector imaging of the right ventricular endocardium of a patient with mitral stenosis. Different colors in figure a. reflect each segment of the right ventricular wall, separately. Each yellow vector in figure b. shows the corresponding myocardial motion towards the reference point at the apex of the heart.

a) 




b)



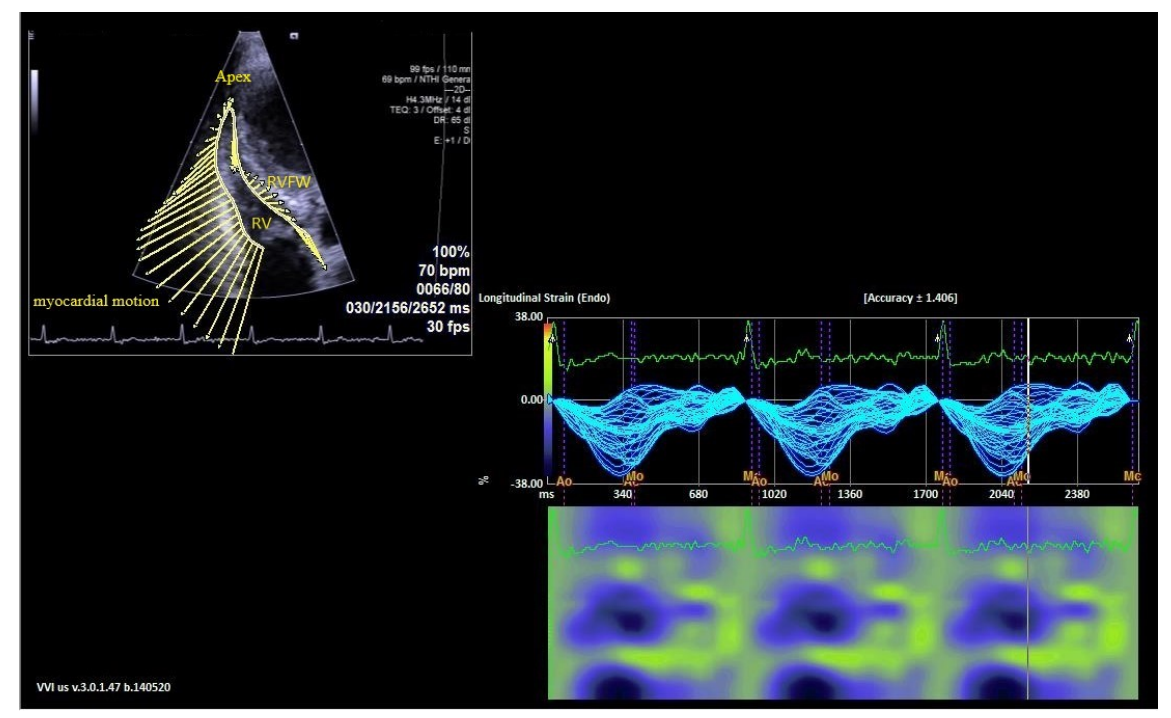

